$X-692-71-493$

PREPRINT

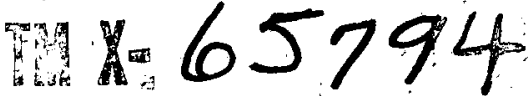

\section{INFLUENCE OF THERMAL ANISOTROPY ON BEST-FIT ESTIMATES OF SHOCK NORMALS}

\section{R. P. LEPPING}

DECEMBER 1971

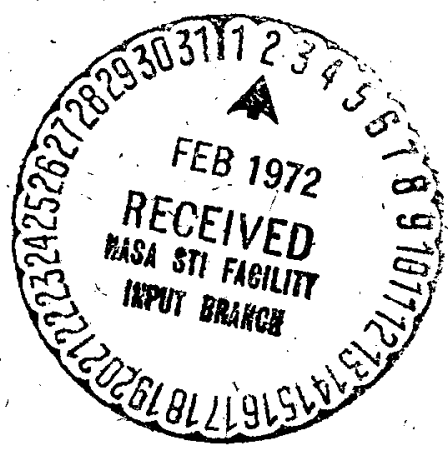

(NASA-TE-X-65794) INFLOENCE OF THERHAL

ANISCRBOEY ON EEST-FIT ESTIMATES OF SHOCK NORHALS $19 \mathrm{p}$
R.P. Lepping (NASA) Dec. 1971

CSCL 20D

$\left.G 3 / 12 \begin{array}{c}\text { Unclas } \\ 13598\end{array}\right)$


INFLUENCE OF THERMAL ANISOTROPY ON BEST-FIT ESTIMATES OF SHOCK NORMALS

by

R. P. Lepping

Laboratory for Extraterrestrial Physics

NASA-Goddard Space Flight Center

Greenbelt, Maryland

December, 1971

Short Title: Effect of Anisotropy on Shock Normals

To be submitted to the J. Geophys. Res. as a Brief Report. 


\section{Abstract}

This paper deals with the influence of thermal anisotropy on the estimates of interplanetary shock parameters and the associated normals and presents a practical theorem for quantitatively correcting for anisotropic effects by weighting the before and after magnetic fields by the same "anisotropy parameter" $h$. The cuantity $h$ depends only on the thermal anisotropies before and after the shock and on the angles between the magnetic fields and the shock normal. It is shown that for fast shocks and for a liberal range of realistic conditions the auantity $h$ lies in the range $0.90<\mathrm{h}<1.22$; this includes pre-shock anisotropy factors as low as $\xi_{1}=0.5$. The theorem can also be applied to most slow shocks but in those cases h usually should be lower, and sometimes markedly lower, than unity. The previously studied fast shock of January 26, 1968, from plasma and magnetic field measurements made on Explorers 33 and 35 , is reexamined in light of this field-weighting theorem. Even for the extreme values of $h$ given above little change results in the shock parameters or in the shock normal, which over the full range of $h$ is estimated to deflect by only $1.7^{\circ}$ for Explorer 33 and $2.0^{\circ}$ for Explorer 35. In no case of the recalculated normal did it deflect more than $1.5^{\circ}$ from the $h=1$ normal, for either spacecraft. These deflections are well within the (95\% certainty) error cone angle, which was $7.6^{\circ}$ in this case. 


\section{Introduction}

This study explores the question of the influence of solar wind therma1 anisotropy on least squares estimates of interplanetary shock parameters and normals, using a select subset of the eight Rankine-Hogoniot conservation eauations. The six equation subset is chosen to avoid using those ecuations which explicitly involve thermal pressure (or temperature), i.e. except for the dependence of the aniosotropy factors on such cuantities. In this manner the normal momentum flux equation and the least well understood of the conservation eauations, the energy flux equation, are not used in the best fit analysis. Other perhaps enually important reasons may be present to justify such a truncation of the full set of eouations (Lepping, 1971). To simplify matters further it is common to use this subset of ecuations expressed for an isotropic medium, that is for $\xi_{1}, \xi_{2}=1$, where

$$
\xi_{i}=1-\frac{4 \Pi\left(P_{\mu}-P_{\perp}\right)_{i}}{B_{i}^{2}}
$$

and where the subscripts refer to before (1) and after (2) the shock, and $P_{\|}$and $P_{\perp}$ are the thermal pressures (ion plus electron) parallel and perpendicular to the magnetic field direction. Then the new subset of equations is independent of thermal pressure. This approximation was employed by Ogilvie and Burlaga (1969), Chao (1970), and Lepping and Argentiero (1971).

This paper is also restricted to the consideration of only the six enuation subset but allows for non-unity anisotropy factors. These more general ecuations, cast in a shock frame of reference, are as follows 


$$
\begin{aligned}
& {\left[\rho U_{n}\right]_{1}^{2}=0} \\
& {\left[\rho U_{n} U_{t}-\xi B_{n} B_{t} / 4 \pi\right]_{1}^{2}=0} \\
& {\left[U_{n} B_{t}-U_{t} B_{n}\right]_{1}^{2}=0}
\end{aligned}
$$

where $t=t_{1}$ or $t_{2}$ and

$$
\left[\mathrm{B}_{\mathrm{n}}\right]_{1}^{2}=0
$$

where $\rho$ is the plasma mass density, $U_{n}$ is the plasma bulk velocity component normal to the shock surface, $U_{t}\left(t=t_{1}\right.$ or $\left.t_{2}\right)$ are the velocity components tangential to the shock surface, $B_{n}$ and $B_{t}\left(t=t_{1}\right.$ or $\left.t_{2}\right)$ are the associated normal and tangential components of the magnetic field. The symbol $[\mathrm{A}]_{1}^{2}=\mathrm{A}_{2}-\mathrm{A}_{1}$ refers to the difference between the pre-shock state $\left(A_{1}\right)$ and post-shock state $\left(A_{2}\right)$. Notice only equations (3) and (4) contain the anisotropy factors $\xi_{1}$ and $\xi_{2}$. Generalization of the Problem

Guided by the magnetic field coplanarity theorem, which has been shown independently by several investigators to hold for the general case of an anisotropic medium (Hudson, 1970, Neubauer, 1970 and Chao, 1970), we use the following identifications for the shock front tangential and normal unit vectors (Lepping and Argentiero, 1970):

$$
\begin{aligned}
& \vec{t}_{1}=\frac{\overrightarrow{\Delta B}}{|\overrightarrow{\Delta B}|} \\
& \vec{t}_{2}=\frac{\vec{B}_{1} \times \vec{B}_{2}}{\left|\vec{B}_{1} \times \vec{B}_{2}\right|} \\
& \vec{n}=\vec{t}_{1} \times \vec{t}_{2}
\end{aligned}
$$


where $\quad \overrightarrow{\Delta B} \equiv \overrightarrow{B_{2}}-\overrightarrow{B_{1}}$.

These are used along with the transformation equation

$$
\vec{U}_{i}=\vec{v}_{i}-\vec{v}_{s}(i=1,2)
$$

in order to separate equations (2) to (7) into two convenient sets, three equations each. Notice that $\vec{V}_{s}$ is the local shock velocity as measured in some "fixed" frame of reference, such as an inertial system at rest with respect to the sun. Then $\vec{V}_{i}$ is the bulk plasma velocity measured in the same reference system.

These two sets of three equations each are the following:

a). the shock velocity set

$$
\vec{V}_{s}=V_{s} \vec{n}
$$

where the shock normal is given by

$$
\vec{n}=\frac{\overrightarrow{\Delta B} \times\left(\vec{B}_{1} \times \vec{B}_{2}\right)}{\left|\overrightarrow{\Delta B} \times\left(\vec{B}_{1} \times \vec{B}_{2}\right)\right|}
$$

from $(8-10)$ [Note that $\vec{t}_{1} \cdot \vec{t}_{2}=0$ ], and the shock speed is

$$
V_{s}=\frac{\rho_{2} \vec{W} \cdot \vec{n}}{\rho_{2}-\rho_{1}}+\vec{v}_{1} \cdot \vec{n}
$$

where

$$
\overrightarrow{\mathrm{W}} \equiv \overrightarrow{\mathrm{V}}_{2}-\overrightarrow{\mathrm{V}}_{1} \text {. }
$$

b). the general overdetermination equations

$$
\begin{aligned}
& \vec{W} \cdot \vec{S}=0 \\
& \left(\frac{\rho_{2}}{\rho_{1}} \vec{B}_{1}-\vec{B}_{2}\right) \cdot(\vec{W} \times \vec{S})=0 \\
& {\left[\frac{\rho_{1} \rho_{2}}{\rho_{2}-\rho_{1}} \vec{W}(\vec{W} \cdot \overrightarrow{\Delta B})+\frac{\vec{B}_{1} Q}{4 \pi}\right] \cdot(\overrightarrow{\Delta B} \times \vec{S})=0}
\end{aligned}
$$


where $\quad \overrightarrow{\mathrm{S}} \equiv \overrightarrow{\mathrm{B}}_{1} \times \overrightarrow{\mathrm{B}}_{2}$

and

$$
Q \equiv \xi_{1} B_{1}^{2}+\xi_{2} B_{2}^{2}-\left(\xi_{1}+\xi_{2}\right)\left(\vec{B}_{1} \cdot \vec{B}_{2}\right)
$$

For the isotropic case, where $\xi_{1}$ and $\xi_{2}$ are equal to unity, $Q$ becomes $|\overline{\Delta B}|^{2}$ and equation (19) becomes

$$
\left[\frac{\rho_{1} \rho_{2}}{\rho_{2}-\rho_{1}} \vec{W}(\vec{W} \cdot \overrightarrow{\Delta B})+\frac{\vec{B}_{1}|\overrightarrow{\Delta B}|^{2}}{4 \pi}\right] \cdot(\overrightarrow{\Delta B} \times \vec{S})=0
$$

Equations (17), (18), and (22) are presently used to obtain estimates of the eleven shock parameters $\left(\vec{B}_{1}, \vec{B}_{2}, \vec{W}, \rho_{1}, \rho_{2}\right)$. and, in turn, the associated normal, (14), by using a least squares fit of these measured field and plasma data. Equation (15) then is used, for an average value of $\vec{V}_{1}$ over a reasonable time interval, to obtain the local shock speed.

For the general, i.e., anisotropic, problem the situation is similar except that $\xi_{1}, \xi_{2} \neq 1$, and this affects $Q$ which appears in equation (19). To study this influence we proceed as follows. Let us define

$$
\epsilon \equiv \xi_{2} / \xi_{1}-1
$$

and assume that

$$
(0<) \xi_{1} \leq \xi_{2}
$$

It is expected that on1y under very unusual circumstances will this inequality be violated and there is no observational evidence, in the case of interplanetary shocks ( or in any other case of which the author is aware), for which it is violated. The condition that $\xi_{1}>0$ is equivalent to assuming that the plasma does not experience the firehose instability (Eviator and Shultz, 1970, and Burlaga, 1971).

Using (23) we see that $Q$ can be written

$$
Q=\xi_{1} f|\overrightarrow{\Delta B}|^{2}
$$


where

$$
f \equiv 1+g \epsilon
$$

and where $\quad g \equiv \frac{\vec{B}_{2} \cdot \overrightarrow{\Delta B}}{|\overrightarrow{\Delta B}|^{2}}$.

With the aid of (8) g can be written

$$
g=\frac{\mathrm{B}_{2 t_{1}}}{|\overrightarrow{\Delta B}|}=\frac{1}{1-\frac{\tan \theta_{1}}{\tan \theta_{2}}}
$$

where (7) was used to obtain the second equality and where $\theta_{i}(i=1,2)$ is the angle between $\bar{B}_{i}(i=1,2)$ and the shock normal $\vec{n}_{\text {. Notice that }}$ $g$ does not depend on the magnitude of either $\vec{B}_{1}$ or $\vec{B}_{2}$ but only on their directions relative to $\vec{n}$. From (1) we see that $\xi_{1}$ and $\xi_{2}$ ( and therefore $\varepsilon$ from (23)) depend on the magnitude of $\vec{B}_{1}$ and $\vec{B}_{2}$, respectively. Hence, $Q$ depends on the vector quantities $\vec{B}_{1}$ and $\vec{B}_{2}$.

It will be assumed temporarity that

$$
\epsilon<1,
$$

where from (24) $€ \geq 0$.

We will exclude discussion of the cases of the so-called switch-on shock (where $\theta_{1}=0,0<\theta_{2}<\pi / 2$, and $g=1$ ) or the switch-off shock (where $0<\theta_{1}<\pi / 2, \theta_{2}=0, g=0$ ), which are special cases of fast and slow shocks, respectively, because of their expected nonevolutionary characteristics (Jeffrey and Taniuti, 1964). These special cases have never been observed in interplanetary space and would be difficult to. identify in any case due to the naturally occuring fluctuations around interplanetary shocks. Parallel $(\vec{B} \| \vec{n})$ or perpendicular $(\vec{B} \perp \vec{n})$ shocks will also not be considered here. Then the sign of $g$ depends on whether the oblique shoc: under consideration is fast $(+)$ or slow $(-)$. Temporarily we 
restrict our discussion to oblique fast shocks only. Since $0<\theta_{1}<\theta_{2}<\pi / 2$ in all cases of such shocks, where $\vec{n}$ is defined here in such a way that $\theta_{1}$ and $\theta_{2}$ are acute angles, and since $\epsilon \geq 0$, then $g \epsilon$ is always $>0$, but it is expected to be usually $\ll 1$; i.e. $0 \leq \epsilon<g \epsilon \ll 1$ for most realistic cases. Hence f, from (26), will usually be slightly greater than unity. Since $\xi_{1}$ is usually slightly less than unity, the product $\left(\xi_{1} f\right)$ in (25), which must always be positive by our assumptions, is usually closer to unity than $\xi_{1}$. In fact, for all oblique fast shocks (since $f$ is always $>0$ in those cases) $\xi_{1} f>0$. Then for such shocks we can define a positive real parameter $h$ to be

$$
h \equiv\left(\xi_{1} f\right)^{1 / 2}
$$

which we will refer to as the "anisotropy parameter".

To estimate this parameter we use the results of Ghao and Goldstein (1971) from their application of the $\mathrm{R}-\mathrm{H}$ conservation equations to find $\xi_{1}$ and $\xi_{2}$. [A1so see Hundhausen et al., 1967] They use the entire eight equation set and retain only those values which give reasonable electron temperatures as a by-product of their analysis; this turns out to be sufficiently restrictive. Their method of finding the $\xi^{\prime}$ s from the characteristics of Alfven waves around the shock is probably not as reliable and its results will not be considered here. Taking averages of the $\xi$-values that they obtain for four shocks (two fast and two slow), we find that $\xi_{1}=0.77 \pm 0.10$ and $\xi_{2}=0.80 \pm 0.11$. Hence, we arrive at characteristic $\epsilon^{\prime} s$ between 0.00 and 0.19 , and we retain $\epsilon \cong 0.04$ as a most probable value. Since $\tan \theta_{1 / \tan } \theta_{2} \approx 1 / 3$ is a reasonable characteristic value for this ratio, we find that $f \approx 1.06$, and hence 
$\mathrm{h} \approx 0.90$ using $\xi_{1} \approx 0.77$.

For the remainder of this study, restriction (29), that $\varepsilon<<1$, will no longer hold. It was employed only to facilitate discussion of the most common cases of oblique fast shocks.

The Field Weighting Theorem

We now proceed to show, for fast and slow shocks, the significance of the anisotropy parameter, $h$, and the reason for choosing a 1/2-power functional relation in its definition (30). Note that (30) will hold for slow shocks, provided $f>0$. If $f<0$ then $h$ becomes imaginary and (22)' below changes structure (second term assumes an explicit negative sign). Hence, the following theorem fails for slow shocks with $f<0$. Such cases can be easily handled by a simple modification of the theorem. We begin by defining an effective magnetic field $\vec{H}_{i}(i=1,2)$ by

$$
\overrightarrow{\mathrm{H}}_{i}=\mathrm{h} \overrightarrow{\mathrm{B}}_{i}(i=1,2) \text { 。 }
$$

That is, it is the true magnetic field multiplied by the weight factor $h$, which as we have seen contains the effects of the pressure anisotropy and the influence of the shock normal's direction through $\theta_{1}$ and $\theta_{2}$. By multiplying the general equations (17), (18), and (19) by $h^{2}, h^{3}$, and $h^{4}$, respectively, and using the defining equations (25), (30), and (31) we obtain a new set of overdetermination equations, which are identical to the "isotropic" equations (17), (18), and (22) except now $\vec{B}_{i}$ is replaced by $\vec{H}_{i}(i=1,2)$ and the new quantities $\overrightarrow{\mathrm{S}}$ and $\overrightarrow{\Delta \mathrm{H}}$ become $\overrightarrow{\mathrm{S}}=\overrightarrow{\mathrm{H}}_{1} \times \overrightarrow{\mathrm{H}}_{2}$ and $\overrightarrow{\Delta \mathrm{H}}=\overrightarrow{\mathrm{H}}_{2}-\overrightarrow{\mathrm{H}}_{1}$. Also multiplying top and bottom of the right side of equation' (14) by $h^{3}$ yields, in the same manner, an identical equation for $\vec{n}$ except in terms of $\overrightarrow{\mathrm{H}}^{\prime} \mathrm{s}$ instead of $\overrightarrow{\mathrm{B}}^{\prime} \mathrm{s}$. Equations (13), (15), and (16) remain unchanged. Henceforth, for future reference equations (14), (17), (18), 
and (22) in the $\overrightarrow{\mathrm{H}}$-domain will be designated as (14)', (17)', (18)' and (22)'. So by considering the effective field $\vec{H}_{i}$ instead of the real field $\vec{B}_{i}$ our general six-equation set (i.e. including anisotropy) transforms into the same functional forms previously used in the isotropic case, and thus the expression for the normal, (14)', is also formally identical for the isotropic and anisotropic cases. The latter result is, of course, a trivial consequence of the fact that the magnetic field coplanarity theorem holds for the general anisotropic case. The ability to homologously transform the field by (31) and obtain "anisotropic" equations in the $\overrightarrow{\mathrm{H}}$-domain corresponding identically to the "isotropic" equations in the $\vec{B}$-domain has important practical significance with regard to a least-squares bestfit program of estimating shock normals based on the isotropic equations (Lepping and Argentiero, 1971) for fast shocks, and for slow shocks provided $f>0$. Without modification of such a program, it can be used for those shocks (with $f>0$ ) in whose cases the isotropic approximation does not hold by applying a preconditioned weighting of the $\vec{B}$-field by (31) before use of the program. This we refer to as the Field Weighting Theorem.

In practice it might be necessary to carry out the scheme with $h$ initially set equal to unity (or some other first trial) and obtain a first rough estimate of the shock parameters and the normal. The resulting normal, as well as the various shock parameters, will then reflect the inaccuracy of using an inexact value for $h$ even though (14) and (14)' are formally identical (i.e. even though $\vec{n}$ does not explicitely depend on $h$ ). This inaccuracy stems from the implicit dependence of the field components (as well as the plasma parameters) on the value of $h$ through the least-squares best-fitting process; as $h$ changes the components of 
the $\overrightarrow{\mathrm{H}}^{\prime} \mathrm{s}$ in $(14)^{\prime}$ (or equivalently the $\overrightarrow{\mathrm{B}}^{\prime}$ s in (14)) change

with respect to each other. After this first computation with

a trial $\mathrm{h}$ it is a simple matter to use the resulting

"best-fit" estimates of the quantities $\vec{B}_{1}$ and $\vec{B}_{2}$ along with the estimate of $\vec{n}$ to obtain first "best-fit" estimates of $\theta_{1}$ and $\theta_{2}$. These combined with estimates of $\xi_{1}$ and $\xi_{2}$ (either from an educated guess or through the use of the remaining conservation equations or from reliable direct measurements) enables one to calculate a better "best-fit" estimate of $h$. Then all of the input $\vec{B}$-field data can be altered by multiplication by $h$, as in (31), and the effective field $\vec{H}$ used in a repeated operation of the least-squares process to obtain an updated set of shock parameters. This process can be repeated as often as one wishes, but presently a single iteration beyond $h=h(t r i a l)$ is probably sufficient since the factors $\xi_{1}$ and $\xi_{2}$ are usually estimated only rough $1 \mathrm{y}$. For fast shocks $\mathrm{h}$ is not a very sensitive function of $\frac{\tan \theta_{1}}{\tan \theta_{2}}, \xi_{1}$, and $\xi_{2}$ provided $\xi_{1} \leq \xi_{2} \leq 1$ and $\xi_{2} \geq 0.8$, as we will show below.

Expected Range of $h$ for Fast Shocks

We now wish to examine for fast shocks the range of $h$, which. from (23), (26), (28), and (30) can be expressed as

$$
\begin{aligned}
h & =\left(\frac{\xi_{2}-\xi_{1} R}{1-R}\right) 1 / 2 \\
\text { where } & R \equiv \frac{\tan \theta_{1}}{\tan \theta_{2}} .
\end{aligned}
$$

First we consider it reasonable to expect unity for an upper bound on $\xi_{2}$, and retain $\xi_{1} \leq \xi_{2}$. Table 1 a gives $h$ as a function of $\xi_{1}$ for $\xi_{2}=1.0$ and for the cases where $R=\frac{1}{10}, \frac{1}{4}, \frac{1}{3}$, and $\frac{1}{2}$. Notice that for this condition (i。e. $\xi_{2}=1$ ) $h \geq 1$. Tables $1 b$ and $1 c$ give $h$ as a function of

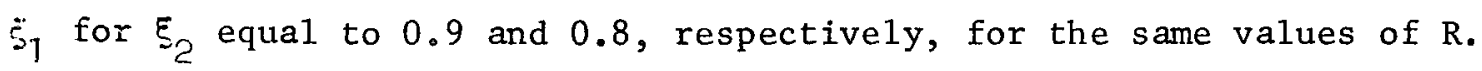


TABLE 1

Values of $h$ for $\xi_{2}=1.0$ (a), 0.9 (b), 0.8 (c) and for $\xi_{1} \geq 0.50$

\begin{tabular}{|c|c|c|c|c|c|}
\hline$\Rightarrow R$ & $\frac{1}{10}$ & $\frac{1}{4}$ & $\frac{1}{3}$ & $\frac{1}{2}$ & \\
\hline 0.50 & 1.027 & 1.080 & 1.118 & 1.225 & (a) \\
\hline 0.75 & 1.014 & 1.041 & 1.061 & 1.118 & $\bar{\xi}_{2}=1.0$ \\
\hline 1.00 & 1.000 & 1.000 & 1.000 & 1.000 & \\
\hline 0.50 & 0.972 & 1.017 & 1.049 & 1.140 & (b) \\
\hline 0.70 & 0.960 & 0.983 & 1.000 & 1.049 & $\xi_{2}=0.9$ \\
\hline 0.90 & 0.949 & 0.949 & 0.949 & 0.949 & \\
\hline 0.50 & 0.913 & 0.949 & 0.975 & 1.049 & (c) \\
\hline 0.65 & 0.904 & 0.922 & 0.935 & 0.975 & $\xi_{2}=0.8$ \\
\hline 0.80 & 0.894 & 0.894 & 0.894 & 0.894 & \\
\hline
\end{tabular}


In, these tables, for intermediate values of $\xi_{1}$ and fixed $R$, values of $\mathrm{h}$ can be 1 inearly interpolated to better than $0.2 \%$ accuracy. For the extreme case of a negligible $R$ (or $R \equiv 0$ ) $h=\sqrt{\xi_{2}}$, independent of $\xi_{1}$, and therefore $h=1.000,0.949$, and 0.894 for $\xi_{2}=1.000,0.900$, and 0.800 , respectively. From an examination of actual interplanetary shocks the value $\mathrm{R}=1 / 2$ appears to be a reasonable arbitrarily chosen upper limit. Many very weak fast shocks are expected to have $R>1 / 2$ but these are difficult to observe. Hence, for these rather extreme ranges of $\xi_{1}$, $\xi_{2}$, and $R$ we see that for fast shocks $h$ is restricted to $h=1.06 \pm 0.17$ provided that $\xi_{I} \leq \xi_{2} \leq 1$ is a reasonable assumption. The value of $h=0.90$ which we have derived from the Chao and Goldstein (1971) results lies at the lower end of this range. Similar tables can, of course, be generated for slow shocks where $h$ would be shown in general to deviate much more markedly from unity due to the fact that $R>1$ for such shocks. Hence, the influence of anisotropy on slow shocks is greater. A Fast Shock Example: January 26, 1968, $\approx 1430 \mathrm{UT}$

The January 26, 1968 fast shock detected by the plasma and magnetic field sensors of Explorers 33 and 35 has been studied in terms of accurate estimates of the shock's parameters and its normal (Lepping, 1971). In that investigation the less general conservation equations, i.e., those for an isotropic medium $\left(\xi_{1}, \xi_{2}=1\right)$, were used in a least-squares bestfit program for the estimation. We show in Tabie 2 the results of the calculations repeated, using the extreme values of $\mathrm{h}$ given above, $\mathrm{h}=0.90$ and $h=1.22$, respectively. The previous results of $h=1.00$ are also listed for comparison. One can readily see that for this characteristic range of $h$, and for both spacecraft observations of the shock, the influence of thermal anisotropy is insignificant with regard to all parameters and sur the normal (given by the components $n_{R}, n_{T}, n_{N}$ ) as well。 In going 
TABLE 2

January 26, 1968 Shock Parameters

Best Fit Values for an 18 Minute Analysis Interval

\begin{tabular}{|c|c|c|c|c|c|c|}
\hline \multirow[t]{2}{*}{ Parameter* } & \multicolumn{3}{|c|}{$\begin{array}{l}\text { Best Fit Value } \\
\text { for Explorer } 33\end{array}$} & \multicolumn{3}{|c|}{$\begin{array}{l}\text { Best Fit Value } \\
\text { for Explorer } 35\end{array}$} \\
\hline & $h=0.90$ & 1.00 & 1.22 & $h=0.90$ & 1.00 & 1.22 \\
\hline $\mathrm{B}_{1 \mathrm{R}}(\gamma)$ & -1.60 & -1.59 & -1.59 & -0.23 & -0.24 & -0.24 \\
\hline $\mathrm{B}_{1 \mathrm{~T}}$ & -3.16 & -3.16 & -3.16 & -3.07 & -3.07 & -3.07 \\
\hline $\mathrm{B}_{1 \mathrm{~N}}$ & -3.49 & -3.49 & -3.49 & -3.83 & -3.83 & -3.84 \\
\hline $\mathrm{B}_{2 \mathrm{R}}$ & -5.13 & -5.15 & -5.20 & -3.39 & -3.41 & -3.43 \\
\hline $\mathrm{B}_{2 \mathrm{~T}}$ & -6.61 & -6.60 & -6.57 & -6.15 & -6.09 & -5.94 \\
\hline $\mathrm{B}_{2 \mathrm{~N}}$ & -7.56 & -7.54 & -7.46 & -8.34 & -8.26 & -8.05 \\
\hline $\mathrm{W}_{\mathrm{R}}(\mathrm{km} / \mathrm{sec})$ & 78.5 & 78.6 & 78.9 & 84.8 & 85.6 & 87.7 \\
\hline $\mathrm{W}_{\mathrm{T}}$ & -38.1 & -37.0 & -34.0 & -35.4 & -35.0 & -34.3 \\
\hline $\mathrm{w}_{\mathrm{N}}$ & -28.6 & -28.3 & -27.7 & -24.4 & -24.2 & -23.7 \\
\hline $\mathrm{N}_{1}\left(\right.$ 非 $\left./ \mathrm{cm}^{3}\right)$ & 4.18 & 4.19 & 4.1 .9 & 4.43 & 4.45 & 4.50 \\
\hline $\mathrm{N}_{2}$ & 9.69 & 9.67 & 9.61 & 10.60 & 10.52 & 10.34 \\
\hline $\mathrm{n}_{\mathrm{R}}$ & 0.828 & 0.826 & 0.821 & 0.854 & 0.850 & 0.838 \\
\hline $\mathrm{n}_{\mathrm{T}}$ & -0.443 & -0.440 & -0.434 & -0.413 & -0.416 & -0.423 \\
\hline $\mathrm{n}_{\mathrm{N}}$ & -0.344 & -0.352 & -0.372 & -0.316 & -0.324 & -0.345 \\
\hline Quality & 1.05 & 1.05 & 1.05 & 1.04 & 1.04 & 1.03 \\
\hline
\end{tabular}

*Vector quantities are given in the $\hat{\mathrm{R}}-\hat{\mathrm{T}}-\hat{\mathrm{N}}$ coordinate system, centered at the spacecraft of interest, such that $\hat{R}$ points away from the sun parallel to the ecliptic plane, $\hat{\mathrm{N}}$ is perpendicular to the plane and "northward", and $\hat{\mathrm{T}}=\hat{\mathrm{N}} x \hat{\mathrm{R}}$ 。 
from $h=0.9$ to $h=1.22$ the normal deflects through $1.7^{\circ}$ for Explorer 33 and $2.0^{\circ}$ for Explorer 35 , and neither recalculated normal deviated more than $1.5^{\circ}$ from the $h=1$ normal, in either spacecraft's case. These deviations are all well within the calculated (95\% certainty) error-cone angle of $7.6^{\circ}$. Since this example is believed to be representative of oblique fast shocks in general (unless $R>1 / 2$ or $\xi_{1}<0.5$ or both), we see that the influence of thermal anisotropy on estimating interplanetary fast shock normals is expected to be negligible, changing it by only a few degrees at most, and the deviations in the shock parameters due to $h \neq 1$ are equally unimportant. It is obvious, for instance, that the differences between the best-fit shock parameters for Explorers 33 and 35 are not accounted for by incorrect anisotropy considerations. More likely these differences are real and are explained by the changes in the field and plasma conditions over distances comparable to the separation of the two spacecraft $(\approx 43.5$ earth radii) and over the associated time delay $(\approx 89 \mathrm{sec})$ of the two observations. Since the two spacecraft at the time of the shock sightings were located in that general vicinity of the earth's bow shock where upstream waves and particles fluxes have been observed, such influences could be consldered as a possible specific explanation of these differences. [The spacecraft were 76.6 (Expl. 33) and 56.9 (Exp1.35) earth radii sunward of the earth during the shock passage.] If actually present, such influences could markedly change the shock parameters from one interplanetary observation point to the other and yet have a negligible effect on the shock front's ability to remain plane on the scale of interest here. However, the condition for expecting these influences to be present, i.e., that the interplanetary magnetic field line (pre-shock field in this case) passing through either spacecraft position be connected to the earth's 
bow shock (Fairfield, 1969), does not hold in this case. Therefore, these differences are more likely related to true interplanetary conditions at that time rather than to phenomena associated with the earth. In table 2 a quality index for the shock estimate is also shown. This index is defined as the square root of the ratio of the total number of points of all shock parameters used in the analysis to the standard $\sigma$-weighted least-squares loss function at convergence (Lepping and Argentiero, 1971). This index is commonly very near to unity for characteristic interplanetary shocks provided reasonable $\sigma$-weights (usually rms deviations) are used in the loss function for all parameters. The table shows that the quality of the estimates is, to three place accuracy, nearly independent of the value of $h$ between 0.90 and 1.22 . The lowest value of 1.03 for $h=1.22$, Explorer 35, suggests (see Table 1a) that a combination such as $\xi_{1} \approx 0.5$ and $\xi_{2} \approx 1.0$ for the January 26,1968 shock is not 1ikely, where $R$ was 0.42 for this case. All that can be safely said here is that $\Delta \xi\left(\equiv_{2}-\xi_{1}\right)$ was probably less than 0.4 or so for this shock on the weak assumption that the overall best fit analysis and model was accurate enough to resolve quality with respect to small changes in $h$. The quality of the estimates of the shock parameters, in most cases, must be insensitive to small changes of $\mathrm{h}$ from unity, and large changes are expected to occur only for slow shocks. Discussion and Conclusions

We have shown, for most realistic fast shocks, that best-fit shock parameters and the associated normals are expected to be only weakly dependent upon thermal anisotropy in the vicinity of the shock. 
[In theoretically estimating pre-shock electron temperatures by using the full Rankine-Hugoniot set of equations Chao (1971) has shown, for a sample set of 18 shocks (11 fast and 7 slow), that such estimates also are only very weakly dependent on the values of $\xi_{1}$ and $\left.\xi_{2}\right]$. Conversely, the restricted subset of equations used in the best-fit procedure are therefore, for fast shocks, unable to effectively resolve the $\xi_{1}$ and $\xi_{2}$ factors even when the normal to the shock is known through other means, for instance geometrical methods. Only reliable higher moment equations, or other independent methods, can be used to ascertain the values of the anisotropy factors. From the point of view of accurately estimating shock normals, however, this is a fortunate circumstance. For slow shocks the lower moment equations should be of greater aid in obtaining $\xi_{1}$ and $\xi_{2}$, but again conversely the normals for these shocks should be less reliably estimated due to their greater dependence on $h$, which is usually not very we11 known from independent sources.

For fast shocks, and slow shocks with $f>0$, when either of whose value of $h$ deviates significantly from unity and provided $\xi_{1}$ and $\xi_{2}$ are estimated with acceptable accuracy, the Field Weighting Theorem should furnish a practical means of improving the estimates of shock normals when the best-fit technique is employed. 


\section{ACKNOWLEDGEMENTS}

I wish to thank Dr. S. Olbert for initiating my interest in this topic and Drs. K. W. Ogilvie, L. F. Burlaga and F. Neubauer for critical comments. I am grateful to Dr. N. F. Ness for helpful remarks and for the magnetic field data, and Dr. J. Binsack for the M.I.T. plasma data, used in this study. 


\section{REFERENCES}

Burlaga, L. F., Hydromagnetic Waves and Discontinuities in the Solar Wind, NASA-X-692-71-95, March 1971. To appear in Space Sci.Rev.

Chao, J. K., Interplanetary Collisionless Shock Waves, M. I. T。 Report No. CSR TR-70-3, February, 1970.

Chao, J. K., Evidence for Waves and/or Turbulence in the Vicinity of Shocks in Space, Proceedings of Cosmic Plasma Physics Conference, ESRIN, Frascati, Italy, September 20-24, 1971.

Chao, J. K. and B. E. Goldstein, Thermal Anisotropies in the Vicinity of Solar Wind Shocks, M. I.T. Report No. CSR-P-71-53, February, 1971.

Eviatar, A。 and M. Schulz, Ion-Temperature Anisotropies and the Structure of the Solar Wind, Planet.Space Sci., 18, 321, 1970.

Fairfield, D. H., Bow Shock Associated Waves Observed in the Far Upstream Interplanetary Medium, J. Geophys. Res., 74, 3541, 1969.

Hudson, P. D., Discontinuities in an Anisotropic Plasma and Their Identification in the Solar Wind, Planet Space Sci., 18, 1611, 1970.

Hundhausen, A. J., S. M. Bame, and N. F. Ness, Solar Wind Thermal Anisotropies: Vela 3 and IMP 3, J. Geophys. Res., 72, 5265, 1967.

Jeffrey, A。 and T. Taninti, Non-Linear Wave Propagation, Mathematics in Science and Engineering Series, Academic Press, New York, 1964.

Lepping, R. P., A Two Spacecraft Test of a Single Spacecraft Method of Estimating Shock Normals, submitted to the Proceedings of the Solar Wind Conference, Asilomar, Pacific Grove, Calif. March 21-26, 1971 also NASA-X-692-71-138, March 1971 .

Lepping, R. P. and P. D. Argentiero, Improved Shock Normals obtained from Combined Magnetic Field and Plasma Data from a Single Spacecraft, NASA-X-692-70-276, July 1970. See esp. Appendix A.

Lepping, R. P. and P. D. Argentiero, Single Spacecraft Method of Estimating Shock Normals, J.Geophys. Res., 76, 4349, 1971.

Neubauer, F. M., Jump Relations for Shock in an Anisotropic Magnetized Plasma, Z. Physik, 237, 205, 1970.

Ogilvie, K. W. and L. F. Burlaga, Hydromagnetic Shocks in the Solar Wind, Solar Phys., 8, 422, 1969. 